\title{
A Novel Fuzzy Friction Compensation Approach to Improve the Performance of a DC Motor Control System
}

\author{
Jason T. Teeter, Mo-yuen Chow, Senior Member, IEEE, and James J. Brickley Jr., Member, IEEE
}

\begin{abstract}
The compensation of friction nonlinearities for servo motor control has received much attention due to undesirable and disturbing effects that the friction often has on conventional control systems. Compensation methods have generally involved selecting a friction model and then using model parameters to cancel the effects of the nonlinearity. In this paper, a method using fuzzy logic for the compensation of nonlinear friction is developed for the control of a dc motor. The method is unique in that a single fuzzy rule is used to compensate directly for the nonlinearity of the physical system. As a result, the method introduces fewer adjustable parameters than a typical fuzzy logic approach while still incorporating many advantages of using fuzzy logic such as the incorporation of heuristic knowledge, ease of implementation and the lack of a need for an accurate mathematical model. The general approach, analysis and experimental results obtained for an actual dc motor system with nonlinear friction characteristics are presented and the effectiveness of the fuzzy friction compensation control technique is discussed. The smoothness of response, response times and disturbance rejection of a PI control system with and without the proposed fuzzy compensator are analyzed and discussed to illustrate the effectiveness of the proposed method.
\end{abstract}

\section{INTRODUCTION}

$\mathbf{T}$ THE phenomenon of friction is common to systems that involve some type of sliding motion [1]. Unmodeled nonlinear friction characteristics are notorious for having undesirable and disturbing effects on conventional controller performance [1]-[4]. The general approach to friction compensation has been to select a friction model and then estimate model parameters [2], [3]. The model is then used in the control scheme to cancel the effects of the friction nonlinearity. Examples of this are by Johnson and Lorenz for obtaining model parameters experimentally and employing the model in both feedforward and feedback control strategies [1]. Canudas et al. propose a control scheme where nonlinear friction effects are compensated adaptively by estimating the parameters of a nonlinear friction model [4]. Although model-based methods have been shown to significantly improve performance, their effectiveness usually depends on the accuracy of the models and their parameters. Model-based robust control methods such as variable-structure control [5] have been developed and applied [6] in order to reduce the sensitivity of a system to parametric uncertainties.

Manuscript received September 11, 1994; revised July 23, 1995. This work was supported by the Electric Power Research Institute, Research Contract RP8004-24: Intelligent Energy Control.

The authors are with the Department of Electrical and Computer Engineering, North Carolina State University, Raleigh, NC 27695-7911, USA.

Publisher Item Identifier S 0278-0046(96)01377-9.
Adaptive and model-free control techniques have become increasingly popular in the control of uncertain and/or strongly nonlinear systems. Artificial neural networks (ANN's) have been successfully applied to the control of many nonlinear and/or time-varying systems [7]. Examples of these applications include using an ANN to learn the plant inverse from experimental input/output pairs [7] and using a parallel ANN to adaptively compensate for changes in plant dynamics [8]. Fuzzy logic has earned appeal in the control systems area as a straightforward method of creating an approximate mathematical representation of human decision-making from general knowledge of the laws which govern a system [9]-[11]. Fuzzy logic controllers (FLC's) have been successfully applied to a variety of problems including water quality control [12], nuclear reactor control [13], motor control [14], and many others. Since FLC's can be developed from heuristic knowledge, the employment of a FL control system often eliminates the need for an accurate mathematical description of the plant to be controlled. Several methods have been proposed to improve the performance of an existing control system by using fuzzy logic. Examples of these include fuzzy tuning of PID controller parameters [15] and fuzzy resetting of control effort for PI-type FLC's [16].

FLC's are typically characterized by a large number of adjustable parameters [9]. This provides flexibility but can also complicate the design process depending on performance requirements and the depth of heuristic knowledge possessed by the designer. If deficiencies in the performance of an existing controller can be attributed to a dominant nonlinearity of the plant, it may be desirable to use a sparse set of fuzzy rules in conjunction with the original controller in order to compensate directly for nonlinearity. The locality-of-control (LOC) quality inherent in fuzzy logic controllers [17] allows the designer to limit the operating range over which the fuzzy rules affect the controller output.

In this paper, a fuzzy friction compensator (FFC) is developed to improve the control of a DC motor control system for which relevant heuristic knowledge of system dynamics is available. The FFC rules are generated by observing system response characteristics over a specified operating range and devising a conceptual strategy for reducing the effects of friction nonlinearities. To demonstrate the effectiveness of the method, a FFC is applied to an actual DC motor for which a PI controller has been designed based on a linear model of the system. The incorporation of the FFC is shown to 
significantly improve performance, particularly in the lowspeed operating region of the motor where the friction effects are most significant.

Since the operating conditions of the motor are used to modify linear controller gains, the compensation method can be viewed as a type of gain scheduling [18]. Gain scheduling has been used extensively in industry for the control of nonlinear systems whose dynamics change with the operating conditions [19]. The method usually involves linearizing a system about several operating points based on the values of auxiliary variables which relate well to system dynamics [18]. Linear controllers are then designed for each point and controller gains are scheduled, or interpolated, during operation. Despite only recent formal treatment of the design and performance of gain-scheduled controllers, scheduling methods have been used successfully to handle parameter variations in complex applications such as flight control [20]. Some potential advantages of using fuzzy logic in a gain scheduling application have been presented by Ling and Edgar [21], although their methods use fuzzy logic for interpolation only. The use of fuzzy logic may enable designers to improve system performance without having to develop system models that accurately predict the effects of nonlinearities.

In Section II, a description of the dc motor system and its inherent nonlinear friction characteristics is presented. In Section III, a PI controller is designed based on a linear, time-invariant model of the system and effects of the friction nonlinearity are discussed. Section IV describes the fuzzy compensation technique used to improve system response and Section $V$ presents experimental results to verify the methodology. The smoothness of response, response times and disturbance rejection of the PI control system with and without the proposed FFC are analyzed and possible extensions of the FFC concept are discussed.

\section{SYSTEM DESCRIPTION}

DC motors are used extensively in industry for applications such as robot arm drives, machine tools, rolling mills, and aircraft control [22], [23]. In this paper, dc motor velocity control is used to illustrate the effectiveness of a proposed fuzzy friction compensator. The motor used in this paper for illustration purposes possesses several nonlinear characteristics including backlash, dead zone and nonlinear friction. Fig. 1 shows the actual steady-state relationship between armature voltage and motor speed obtained by reading input-output pairs as the armature voltage is decreased from rated to zero in the laboratory.

The lowest armature voltage that will prevent the motor from stalling is $4 \mathrm{~V}$. When starting the motor from standstill, an input of at least $4.5 \mathrm{~V}$ is needed to overcome the dead zone and static friction. The nonlinear friction has a significant effect on system dynamics, while the effects of the other nonlinearities are comparatively insignificant. Thus, we focus our attention only on the motor friction in the discussion. Several friction models have been proposed for the analysis of physical systems that involve some type of sliding motion [1]. A popular friction model known as the Stribeck curve [24] is shown in Fig. 1(b).

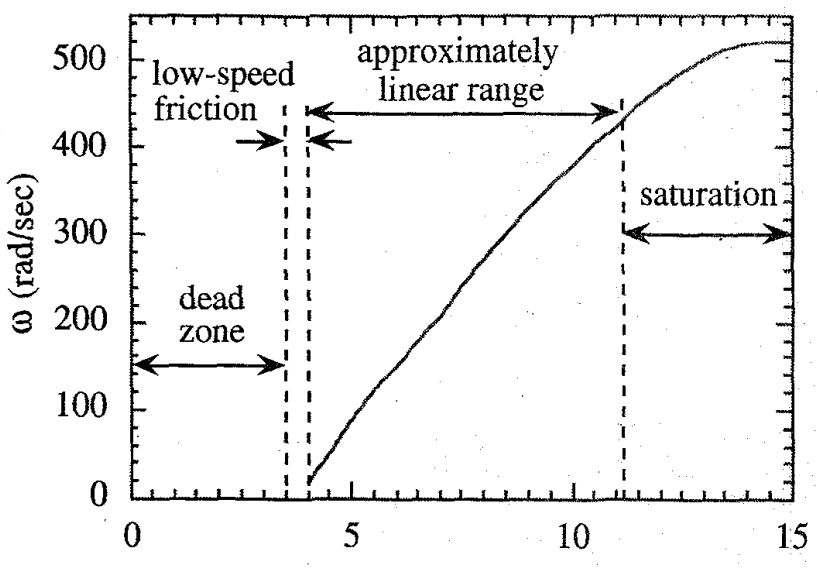

(a)

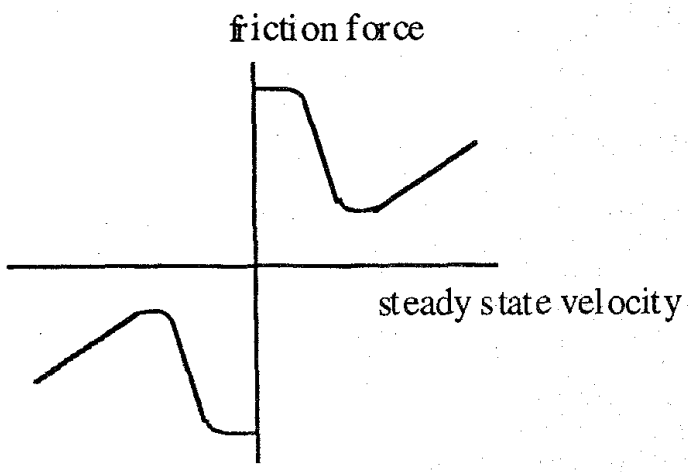

(b)

Fig. 1. (a) DC motor steady-state input-output relationship. (b) Generalized Stribeck curve friction model.

Purely velocity-dependent friction models such as the Stribeck curve do not include additional friction components, such as rising static friction and frictional memory, which may significantly affect performance [24]. Although it may be difficult to obtain an accurate analytic friction model, the qualitative effects of friction are well-known. At low velocities, motion may be intermittent and the resulting "stickslip" phenomenon can lead to overshoot and large-amplitude limit cycles [25]. These effects are discussed in more detail in the following section where they are demonstrated on an actual dc motor. A schematic of the experimental system is shown in Fig. 2(a).

The controllers are implemented on a $486 \mathrm{PC}$ using a LabVIEW graphical programming package: The rotation of the motor shaft generates a tachometer voltage which is then scaled by interfacing electronic circuitry. A National Instruments data acquisition board receives the data via an Analog Devices isolating backplane. After a control value is computed, an output current is generated by the data acquisition board. The current signal passes through the backplane and is then converted to a voltage signal and scaled by the interfacing circuitry before being applied to the armature of the motor. Load disturbances are generated by subjecting a disc on the motor shaft to a magnetic field. The complete system setup is shown in Fig. 2(b) and a close-up view of the motor is given in Fig. 2(c). 


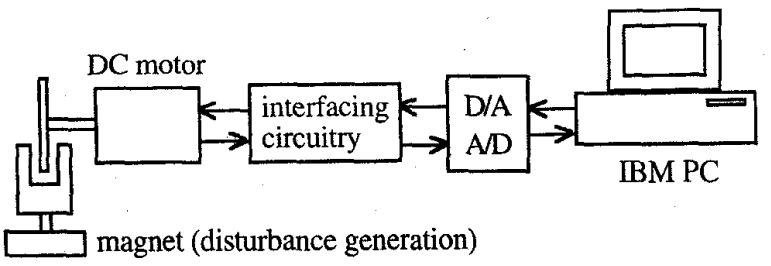

(a)

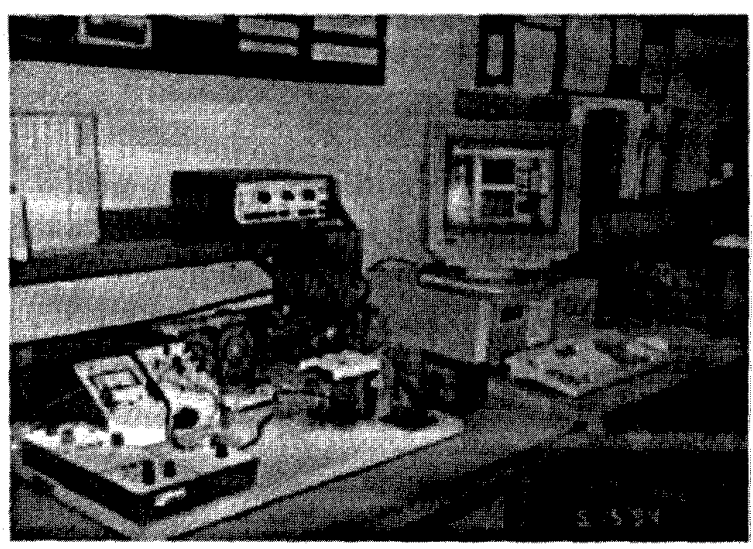

(b)

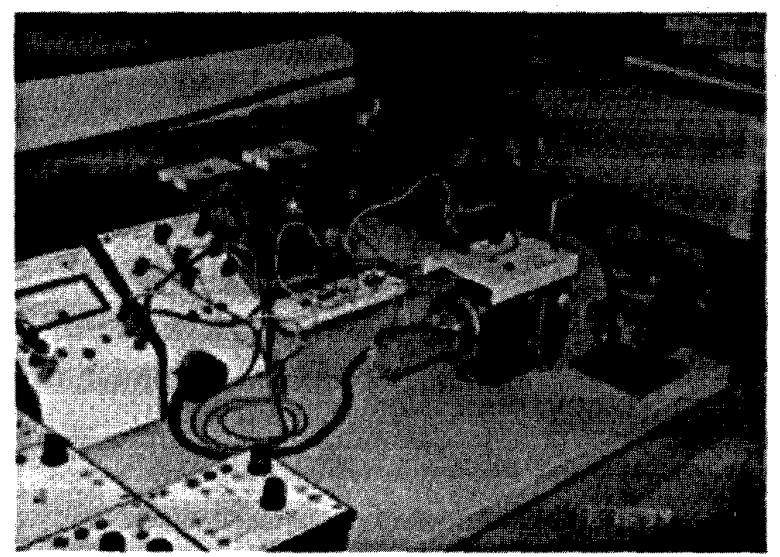

(c)

Fig. 2. (a) Schematic of the experimental dc motor system. (b) Motor control system setup. (c) Close-up view of the dc motor.

\section{PReliminary Controller Design}

In this paper, for illustration purposes, we concentrate on achieving zero steady-state error and smooth, fast response to step inputs and disturbances. These are popular desired motor performance characteristics for many industrial applications. The simplicity and ease of implementation of the PI controller make it a popular choice for many such applications. In order to design a PI controller for our system, we assume that the motor is linear and time-invariant. A second-order model given by

$$
\dot{\mathbf{x}}=\left[\begin{array}{cc}
-\frac{R_{a}}{L_{a}} & -\frac{K_{a}}{L_{a}} \\
\frac{K}{J} & -\frac{f}{J}
\end{array}\right] \mathbf{x}+\left[\begin{array}{c}
\frac{1}{L_{a}} \\
0
\end{array}\right] u
$$

TABLE I

DC MOTOR PARAMETERS

\begin{tabular}{|c|c|}
\hline$R_{a}$ & $4.67 \Omega$ \\
\hline$L_{a}$ & $170 \mathrm{e}-3 \mathrm{H}$ \\
\hline$J$ & $42.6 \mathrm{e}-6 \mathrm{Kg}-\mathrm{m}^{2}$ \\
\hline$f$ & $47.3 \mathrm{e}-6 \mathrm{~N}-\mathrm{m} / \mathrm{rad} / \mathrm{sec}$ \\
\hline$K$ & $14.7 \mathrm{e}-3 \mathrm{~N}-\mathrm{m} / \mathrm{A}$ \\
\hline$K_{b}$ & $14.7 \mathrm{e}-3 \mathrm{~V}-\mathrm{sec} / \mathrm{rad}$ \\
\hline
\end{tabular}

is used where $u$ is the armature voltage, $\mathbf{x}=\left[i_{a} \omega\right]^{T}$, and $i_{a}$ and $\omega$ are the armature current and shaft angular velocity, respectively.

The parameters of the actual motor are estimated via a standard system identification technique based on a linear dc motor model [26]. Their numerical values are listed in Table I. $R_{a}$ and $L_{a}$ are the resistance and the inductance of the motor armature circuit, respectively; $J$ and $f$ are the moment of inertia and the viscous-friction coefficient of the motor and load referred to the motor shaft, respectively; $K$ is the constant relating the armature current to the motor torque, and $K_{b}$ is the constant relating the motor speed to the dc motor's back-emf.

A digital PI controller described by the difference equation

$$
\begin{aligned}
u(k)= & u(k-1)+\left(K_{P}+\frac{K_{I} T_{S}}{2}\right) e(k) \\
& +\left(\frac{K_{I} T_{S}}{2}-K_{P}\right) e(k-1)
\end{aligned}
$$

is designed to provide critically damped step responses and satisfactory response times for a sample period $T_{S}=10 \mathrm{~ms}$. The amplifier for the motor saturates at $15 \mathrm{~V}$, at which point the effectiveness of the feedback loop is broken. When this happens, the output of the controller remains saturated until the error has been negative for a sufficiently long time to allow the magnitude of the integral term to become small. This phenomenon is called integrator windup [27]. To avoid potential problems caused by integrator windup, the following limiting laws are used:

1) $e(-1) \equiv e(0)$.

2) If $u_{\text {calc }}(k)>15$, set $u(k)=15$.

where $u_{\text {calc }}(k)$ represents the control output calculated by the PI control law. The first law prevents the control output from immediately taking on a large value due to the step reference introduced at time $t=0$. The second law is a simple backcalculation algorithm commonly used to avoid windup [27]. The PI controller gains are chosen to be $K_{P}=0.12$ and $K_{I}=0.264$ based on the root locus design method [26].

The practical operating range of our experimental motor is $\omega \in[50450] \mathrm{rad} / \mathrm{s}$. Typical responses of the linear model and the actual system for reference inputs of $100 \mathrm{rad} / \mathrm{sand} 300$ $\mathrm{rad} / \mathrm{s}$ are given in Fig. 3(a) and (b).

It is clear that the friction nonlinearity has an adverse effect on system response, causing overshoot for small reference inputs and oscillatory transient response for large reference inputs. It is reasonable to expect that load disturbances could induce limit cycles due to the nonlinear friction characteristics of the motor. To verify this, we introduce step disturbances at 

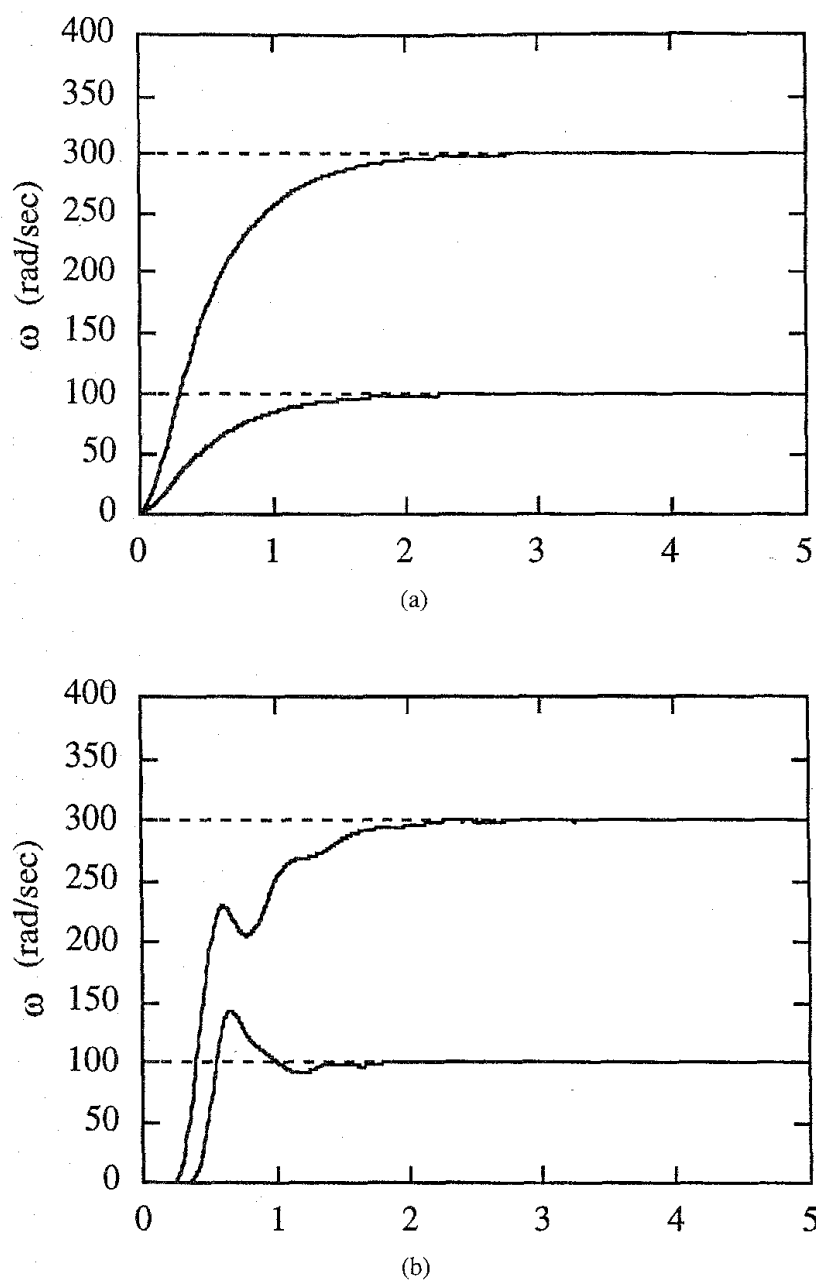

Fig. 3. (a) Typical step responses of the simulated linear dc motor model under PI control. (b) Typical step responses of the actual dc motor system under PI control.

steady-state conditions for the same reference inputs used in Fig. 3(a) and (b). Fig. 4 shows typical system responses under load disturbance.

The load disturbances cause large-amplitude limit cycles, as expected, although this behavior is not limited to small reference inputs. This suggests that the unmodeled friction nonlinearity is significant over a larger operating range than was first believed. In the next section, we present a method of compensating for the friction nonlinearity using fuzzy logic.

\section{FUZZY FRICTION COMPENSATION}

Our objective is to reduce the effects of friction without completely redesigning the controller. The approach taken is to use fuzzy logic to modify the parameters of our original PI controller. A block diagram of the modified system is shown in Fig. 5.

Nonlinear friction effects are less noticeable for responses to large reference inputs since the controller output is less sensitive to large changes in error until the motor speed nears the reference, at which point the nonlinear friction effects are

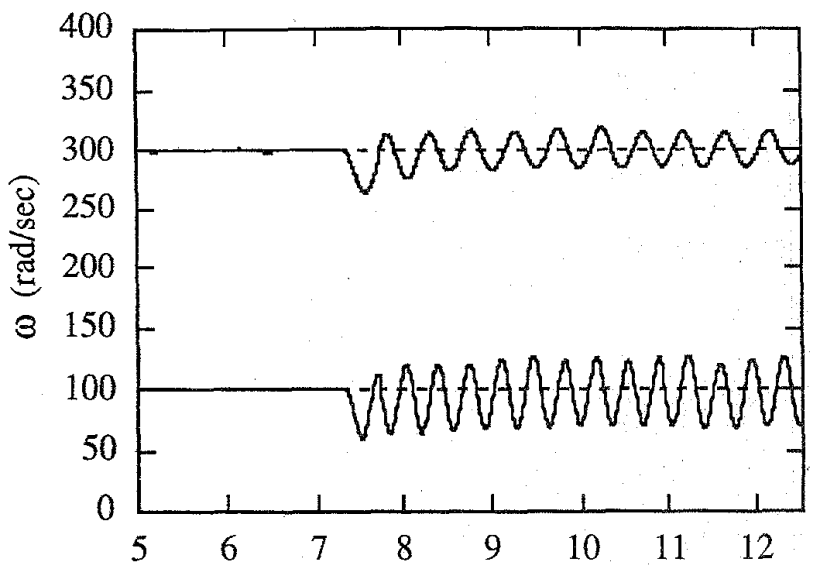

Fig. 4. Typical responses of the actual PI control system under load disturbance.

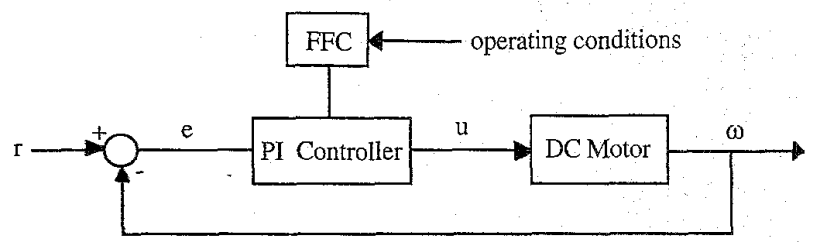

Fig. 5. Block diagram of the PI control system with FFC.

fairly small. For smaller reference inputs, the nonlinearity is significant even when errors are small.

In order to compensate for nonlinear friction effects, our FFC accepts as inputs the controller output, the motor speed and the reference. The linear PI controller designed in Section III is used as a nominal controller while the FFC computes a selected controller gain denoted by $K_{\mathrm{fz}}$. Thus

$$
u(k)=u(k-1)+K_{1} e(k)+K_{2} e(k-1)
$$

becomes

$$
u(k)=u(k-1)+K_{\mathrm{fz}}(r, u, \omega)\left(K_{1} e(k)+K_{2} e(k-1)\right)
$$

where (3) is the equivalent difference equation of the nominal PI controller and $K_{f z}(r, u, \omega)$ in (4) reflects the nonlinear, condition-dependent nature of the computed gain. The FFC is composed of the following rule

$$
\begin{aligned}
& \text { IF } r \text { IS SMALL } \\
& \text { AND } u \text { IS LARGE } \\
& \text { AND } \omega \text { IS SMALL } \\
& \text { THEN DECREASE } K_{\mathrm{fz}}
\end{aligned}
$$

where $r$ is the reference, $u$ is the motor input and $\omega$ is the motor speed.

The rule is derived from heuristic knowledge of the physical system. As the voltage input to the motor increases in response to the step reference input, the static friction and low-speed friction significantly limit the motion of the motor shaft and the motor input continues to increase quickly. The antecedents in (5) involving $u$ and $\omega$ describe the operating conditions of the system immediately before the motor shaft slips and either overshoots the reference at low speeds or oscillates before 


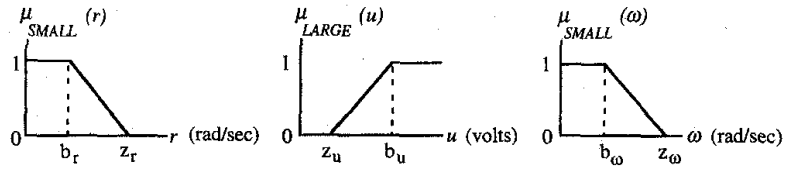

Fig. 6. Fuzzy membership functions for the FFC rule.

approaching the reference at high speeds as shown in Fig. 3(b). The rule consequent serves to limit the change in the motor input when the operating conditions indicate that this behavior is about to occur. The antecedent in (5) involving $r$ provides a method of attenuating the effect of the rule for larger reference inputs in order to compensate for the decreased effects of the nonlinear friction at higher speeds. If this part of the rule were omitted, the system response would be unacceptably slow for large reference inputs.

The membership functions $\{\mu(\cdot)\}$ for the fuzzy rule are shown in Fig. 6. Piecewise-linear functions are used here for the purpose of simplicity; smooth sigmoidal functions could also be used.

Correlation-minimum encoding [28], which yields the minimum fuzzy degree of truth of the rule antecedents, is used to compute the rule output so that

$$
K_{\mathrm{fZ}}=1-0.9 \min \left\{\mu_{\mathrm{SMALL}}(r), \mu_{\mathrm{LARGE}}(u), \mu_{\mathrm{SMALL}}(\omega)\right\}
$$

Note that we have simplified the problem by allowing the FFC to change only $K_{\mathrm{fz}}$ (i.e., the zero of the PI controller is not affected) and restricting the maximum value of $K_{\mathrm{fz}}$ to its nominal value of one. Thus, the FFC compensates for the nonlinear friction by reducing the gain of the controller when the system is most sensitive to the nonlinearity. The 0.9 factor is included in (6) to preserve the integrating operation of the controller so that steady-state tracking error is eliminated provided the system is asymptotically stable. This factor can be adjusted depending on performance specifications; in general, using values closer to 1 increases the influence of the fuzzy rule but results in slower convergence for disturbance responses. Since the FFC output is obtained from only one rule, there is no defuzzification process. If finer control is required or more rigorous performance specifications are given, more rules might be required for the FFC and defuzzification may be necessary [28].

Membership function parameters are tuned off-line according to heuristic rules. Since the friction nonlinearity has the greatest effect at low speeds, initial values of breakpoints $b_{r}$ and $b_{\omega}$ are chosen to be relatively large so that $\mu(r)=$ $\mu(\omega)=1$ at each time step for responses to small reference inputs. Parameters $b_{u}$ and $z_{u}$ are then adjusted to obtain the best performance for these inputs. Thus, emphasis is placed on improving performance in the low-speed operating range of the system where the effects of the friction nonlinearity are most significant. Intercept $z_{u}$ is constrained by $z_{u}<u_{s}$, where $u_{s}$ is the minimum steady-state motor input required to overcome the static friction. This is done so that the motor input can approach $u_{s}$ quickly but the fuzzy rule affects the input rate before the motor shaft slips.

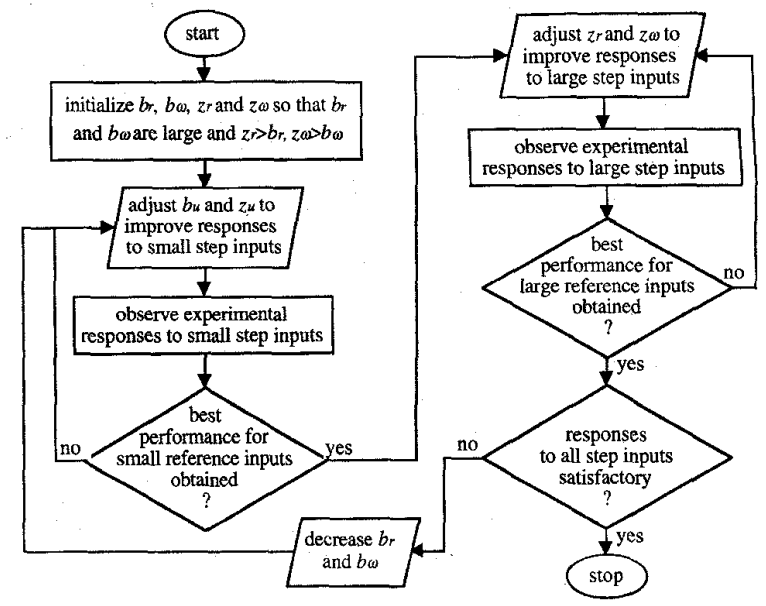

Fig. 7. Flowchart of the parameter modification process.

Next, $z_{r}$ and $z_{\omega}$ are adjusted to obtain the best performance for reference inputs in the remainder of the operating range. Since step reference inputs are used, adjusting $z_{r}$ for a specific reference input value causes the motor input rate to be adjusted equally each time step the value of $\mu_{\mathrm{SMALL}}(r)$ is the minimum of the set in (6). Adjusting $z_{\omega}$, however, results in changes in the input rate which depend on the output at each time step. Thus, $z_{r}$ can be adjusted to affect performance over a range of reference inputs while $z_{\omega}$ can be adjusted to affect performance in a certain operating region for each response. Once these parameters have been tuned, $b_{r}$ and $b_{\omega}$ are adjusted and the entire process is repeated until the best overall performance is obtained. A flowchart of the parameter modification process is given in Fig. 7.

As discussed in the introduction, the role of the FFC resembles that of a gain scheduler. Conceptually, we can view the FFC as computing the controller gain based on the input and output of the system and scheduling the gain based on the reference. Friction compensation and gain interpolation are implicit since the gain is computed at each time step by the fuzzy logic and heuristic knowledge of the nonlinear motor dynamics is used to choose membership function parameters.

\section{FUZZY FRICTION COMPENSATOR RESULTS}

The final parameters chosen for the membership functions of the FFC rule are

$$
\begin{aligned}
\left\{b_{r}, b_{u}, b_{\omega}\right\} & =\{200,6,100\} \\
\left\{z_{r}, z_{u}, z_{\omega}\right\} & =\{600,2,600\}
\end{aligned} .
$$

Note that $z_{r}=z_{\omega}=600$ even though the maximum attainable speed of our motor is about $500 \mathrm{rad} / \mathrm{s}$. The parameters in (7) describe the shapes of the membership functions over the range of attainable values for each variable; it is not required that the parameters themselves lie in these ranges. Fig. 8 shows typical responses of the PI control system with FFC for reference inputs of $100 \mathrm{rad} / \mathrm{s}$ and $300 \mathrm{rad} / \mathrm{s}$. The step responses of the original system as shown in Fig. 3(b) are included for the purpose of comparison. 


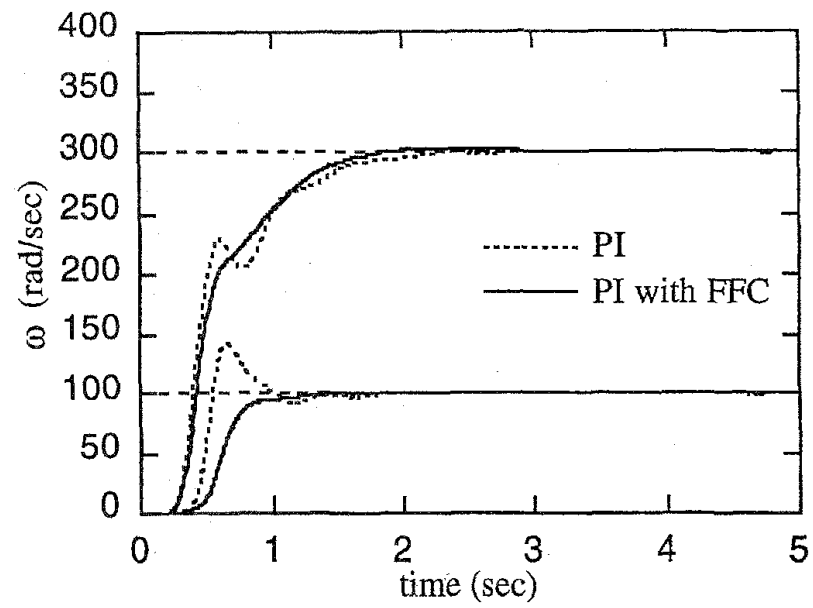

Fig. 8. Typical step responses of the actual system with and without FFC.

The use of fuzzy friction compensation results in significantly smoother step responses with response times comparable to those of the original system. It should be noted that smooth response can be obtained by decreasing the constant gains of the original PI controller, but the resulting increase in response time is unacceptably large. In order to objectively evaluate the performance of the system with and without the FFC, we employ the following cost function for each step response

$$
J=a_{1} J_{1}+a_{2} J_{2}
$$

where

$$
\begin{aligned}
& J_{1}=\sum_{k=0}^{N} e(k)^{2} \\
& J_{2}=\sum_{i=1}^{M} \Delta e_{b}(i)^{2}
\end{aligned}
$$

and

$$
\left\{\Delta e_{b}(i)\right\}=\{\Delta e(k) \mid e(k) \Delta e(k)>0\} .
$$

$J_{1}$ places a penalty on both response time and poor convergence. $J_{2}$ places an extra penalty on overshoot, undershoot and oscillatory behavior. In the event that two responses to the same reference input are critically damped or overdamped so that $J_{2}=0$, the fastest response will always yield the smallest cost. On the other hand, a poorly damped response may yield a smaller cost if it is sufficiently faster than a smooth response. Since the feedback control system is inherently nonlinear, it is difficult to compare performance analytically. In this paper, we present experimental results in order to support the improvement in performance of the FFC feedback control law over that of the conventional PI controller.

A total of 100 experimental trials are performed with and without the FFC using randomly generated reference inputs between $50 \mathrm{rad} / \mathrm{s}$ and $450 \mathrm{rad} / \mathrm{s}$. The number of trials is chosen so that the conclusions drawn are statistically significant [29]. Vectors of the costs in (9) and (10) are formed so that

$$
\begin{aligned}
& \mathbf{J}_{1}=\left[J_{1}\left(r_{1}\right), J_{1}\left(r_{2}\right), \ldots, J_{1}\left(r_{200}\right)\right]^{T} \in \Re^{200 \times 1}, \\
& J_{2}=\left[J_{2}\left(r_{1}\right), J_{2}\left(r_{2}\right), \ldots, J_{2}\left(r_{200}\right)\right]^{T} \in \Re^{200 \times 1}
\end{aligned}
$$

then (12) and (13) are normalized so that

$$
\begin{aligned}
& \hat{\mathbf{J}}_{1}=\mathbf{J}_{1} /\left\|\mathbf{J}_{1}\right\|_{\infty}, \\
& \hat{\mathbf{J}}_{2}=\mathbf{J}_{2} /\left\|\mathbf{J}_{2}\right\|_{\infty} .
\end{aligned}
$$

The vector of total costs is given by

$$
\hat{\mathbf{J}}=c_{1} \hat{\mathbf{J}}_{1}+c_{2} \hat{\mathbf{J}}_{2},
$$

so that the values of $c_{1}$ and $c_{2}$ represent the relative contributions of (14) and (15) to the total cost for each reference. The final time for each reference is chosen to be 5 seconds since all observed responses settle before this time. Fig. 9(a) and (b) shows the costs of (14) and (15), respectively, for the system with and without FFC.

The cost curve of Fig. 9(b) for the system without the FFC can be divided into three regions that illustrate how the undesirable friction effects decrease as the reference is increased. In region I, the oscillatory behavior caused by the nonlinear friction results in a significant and roughly constant value for $J_{2}$. In region II, the nonlinear friction effects decrease and $J_{2}$ decreases roughly linearly as the reference increases. In region III, the nonlinear friction has a relatively insignificant effect on step response and the effects of incorporating the FFC are least noticeable.

From Fig. 9(a) and (b) it is evident that the FFC effectively reduces the values of $J_{2}$ for references in regions. I and II without significantly increasing response times. Thus, almost any combination of cost function weights will result in a larger total cost corresponding to the system without FFC. As an example, cost function weights are chosen to be $\left\{c_{1}, c_{2}\right\}=$ $\{0.7,0.3\}$ so that primary importance is placed on speed of response but oscillatory behavior is still sufficiently penalized. Total costs given by (16) for each reference are plotted in Fig. 9(c) along with the regions shown in Fig. 9(b).

The friction that causes poor step response is also the main cause of disturbance-induced limit cycles. Thus, we expect the incorporation of the FFC to improve the disturbance response of the system. Since the original system is prone to limit cycles, we investigate the stability of the system rather than the transient response. In our discussion, the system is deemed "stable" if the tracking error caused by the disturbance approaches zero asymptotically provided the input does not saturate. To illustrate the effects of the FFC, step disturbances equivalent to those used for the PI control system are introduced at steady-state conditions for reference inputs of $100 \mathrm{rad} / \mathrm{s}$ and $300 \mathrm{rad} / \mathrm{s}$. Fig. 10(a) shows typical responses of the system with FFC under load disturbance. Incorporation of the FFC results in asymptotically stable disturbance rejection for both reference inputs.

It should be noted that here the FFC parameters are chosen based on the performance of system step response. The FFC improves the response under load disturbance because the friction nonlinearity that causes poor system step response is also responsible for limit cycles under load disturbance. If the response of the system under changing loads were more important than the step response, the FFC rules and parameters could be adjusted accordingly either off-line by a human operator or on-line via an adaptive method. For 


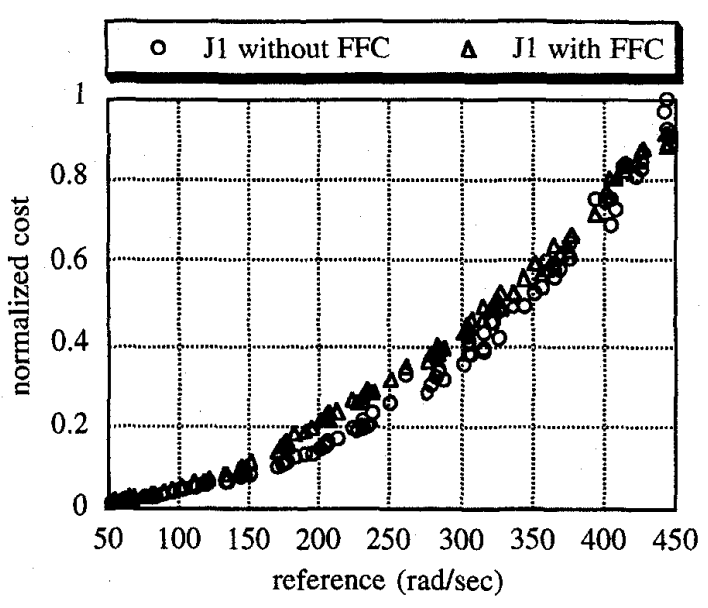

(a)

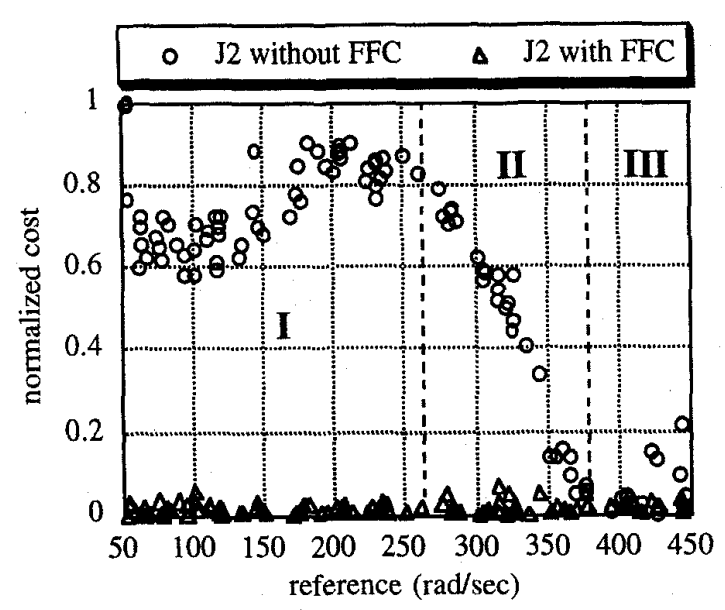

(b)

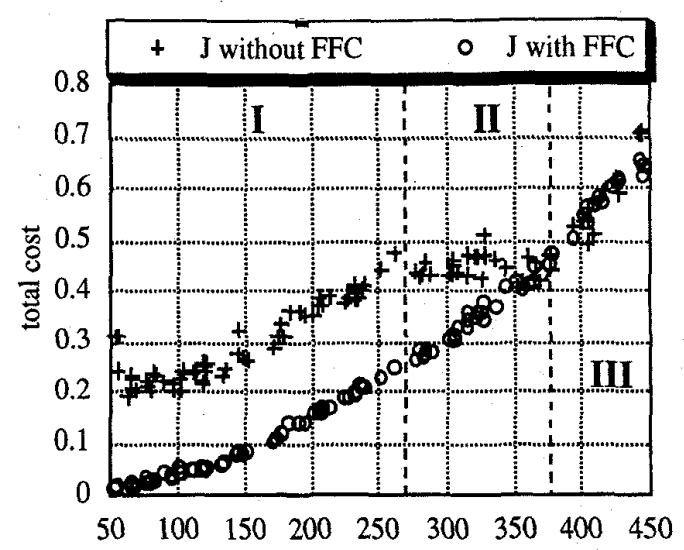

(c)

Fig. 9. (a) Normalized $J_{1}$ for the actual system with and without FFC. (b) Normalized $J_{2}$ for the actual system with and without FFC. (c) Total step response costs for the actual system with and without FFC.

example, the antecedent in (5) involving $u$ could be modified. The steady-state control voltage corresponding to a particular reference speed depends on the amount of applied load torque,

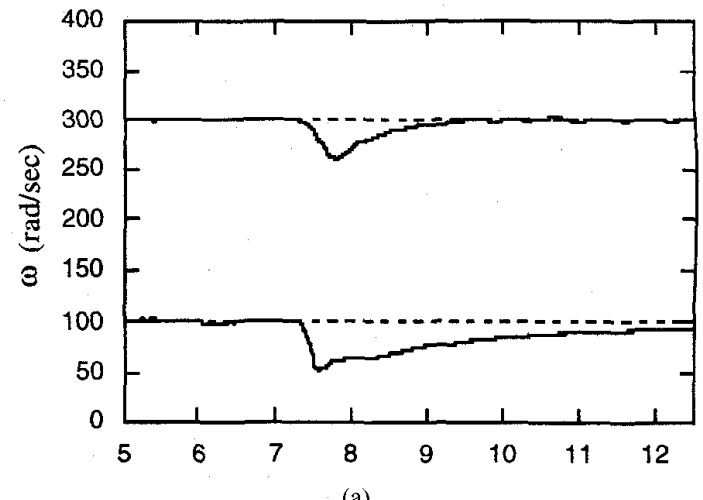

(a)

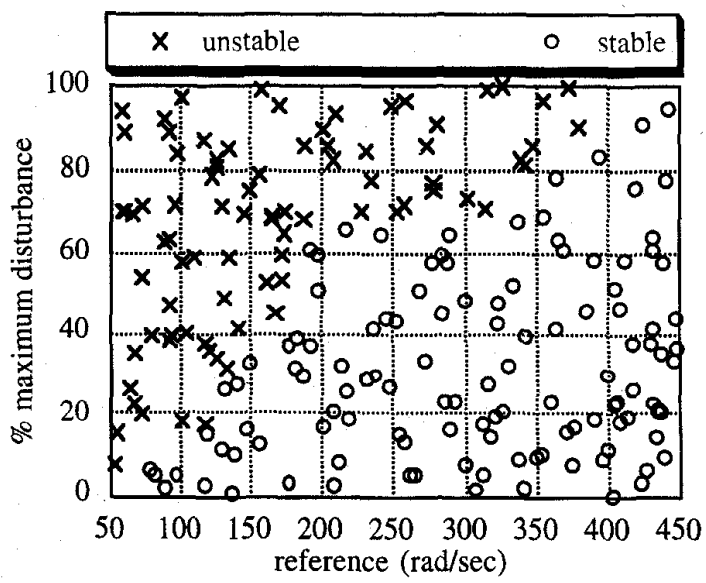

(b)

Fig. 10. (a) Typical responses of the actual system with FFC underload disturbance. (b) Stability region for the actual system without FFC.

so computing controller parameters based on absolute values of $u$ may result in undesirably slow disturbance rejection. This effect can be seen in the response in Fig. 10(a) corresponding to a reference speed of $100 \mathrm{rad} / \mathrm{s}$. One potential solution would be to substitute an expression for $u$ in (5) which depends on $u$ but decreases as the response converges. This would provide another means of incorporating measurable operating conditions into the FFC without making it significantly more complex.

Due to the nonlinearities of the motor and controller, experimental results are used once again to evaluate system performance. In order to illustrate the effects of the FFC on the disturbance response for a representative number of references and disturbances, 200 experimental trials are performed using randomly generated reference/disturbance pairs. Again, the number of trials is chosen so that the conclusions drawn are statistically significant. Reference inputs are between 50 $\mathrm{rad} / \mathrm{s}$ and $450 \mathrm{rad} / \mathrm{s}$ and step disturbances are between $0 \%$ and $1.00 \%$ of maximum for each reference. Each "maximum" disturbance value corresponds to the largest value that can be applied without causing a saturated steady-state control output. The addition of the FFC results in stable disturbance response for all reference/disturbance pairs tested. Fig. 10(b) presents the stability region for the original system. It is evident that 
incorporation of the FFC results in a significantly larger region of stability for disturbance responses.

\section{CONCLUSION}

A novel method of friction compensation using fuzzy logic has been shown to significantly improve the performance of a dc motor system with nonlinear friction characteristics. While the method requires heuristic knowledge of system dynamics and lacks the mathematical justification that may accompany model-based methods, the ability to compute controller parameters based on simple heuristic rules makes it attractive in situations where mathematical modeling is not feasible. The FFC method presented here is still somewhat of an art, which is generally true for the design of fuzzy controllers. The results presented here support the popular view that fuzzy logic has a promising future in the design and implementation of automatic control systems.

\section{REFERENCES}

[1] C. T. Johnson and R. D. Lorenz, "Experimental identification of friction and its compensation in precise, position controlled mechanisms," IEEE Trans Ind. Applicat., vol. 28, no. 6, pp. 1392-1398, 1992.

[2] C. D. Walrath, "Adaptive gearing friction compensation based on recent knowledge of dynamic friction," Automatica, vol. 20, pp. 717-727, 1984.

[3] S. C. Gomes and J. P. Chretien, "Dynamic modeling and friction compensated control of a robot manipulator joint," in Proc IEEE Int. Conf. Robot., Automat., 1992, pp. 1429-1435.

[4] C. Canudas, K. J. Astrom, and K. Braun, "Adaptive friction compensation in DC-motor drives," IEEE J Robot, Automat, vol. RA-3, no. 6, pp. 681-685, 1987.

[5] J. Y. Hung, W. Gao, and J. C. Hung, "Variable structure control: A survey," IEEE Trans Ind. Electron., vol. 40, no. 1, pp. 2-22, 1993.

[6] G. S. Buja, R. Menis, and M. I. Valla; "Variable structure control of an SRM drive," IEEE Trans. Ind. Electron., vol. 40, no. 1, pp. 56-63, 1993.

[7] T.-S. Low, T.-H. Lee, and H.-K. Lim, "A methodology for neural network training for control of drives with nonlinearities," IEEE Trans Ind Electron. vol. 39, no. 2, pp. 243-249, 1993.

[8] T.-H. Lee, W. K. Tan, and M. H. Ang Jr., "A neural network control system with parallel adaptive enhancements applicable to nonlinear servomechanisms," IEEE Trans Ind Electron., vol. 41, no. 3, pp. 269-277, 1994.

[9] C. C. Lee, "Fuzzy logic in control systems: Fuzzy logic controller-Parts I, II," IEEE Trans. Syst., Man, Cybern., vol. 20, no. 2, pp. 404-435, 1990

[10] B. M. Bose, "Expert system, fuzzy logic, and neural network applications in power electronics and motion control," in Proc IEEE, 1994, vol. 82 , no. 8 , pp. $1303-1323$.

[11] R. Sharpe and M. Chow, "A methodology using fuzzy logic to optimize feedforward artificial neural network configurations," IEEE Trans Syst., Man, Cybern., vol. 24, no. 5, pp. 760-768, 1994.

[12] O. Yagashita, O. Itoh, and M. Sugeno, "Application of fuzzy reasoning to the water purification process," Ind. Applicat. Fuzzy Contr., M. Sugeno, Ed., North-Holland, Amsterdam, pp. 19-40, 1985.

[13] M. Kinoshita, T. Fukuzaki, T. Satoh, and M. Miyake, "An automatic operation method for control rods in BWR plants," in Proc. Specialists' Meeting on In-core Instrumentation and Reactor Core Assessment, Cadarache; France, 1988.

[14] M. Chow and A. Menozzi, "On the comparison of emerging and conventional techniques for DC motor control," in Proc. IECON, 1993.

[15] S. He, S. Tan, F. Xu, and P. Wang, "Fuzzy self-tuning of PID controllers," Fuzzy Sets and Systems, vol. 56, no. 1, pp. 37-46, 1993.

[16] J. Lee, "On methods for improving performance of PI-type fuzzy logic controllers," IEEE Trans. Fuzzy Syst., vol. 1, no. 4, pp. 298-301, 1993.

[17] D. E. Thomas and B. Armstrong-Helouvry, "Fuzzy logic control-A taxonomy of demonstrated benefits," in Proc. IEEE, 1995, vol. 83, no. 3, pp. $407-421$

[18] K. J. Astrom and B. Wittenmark, Adaptive Control. Reading, MA: Addison-Wesley, 1989.

[19] W. J. Rugh, "Analytical framework for gain scheduling," IEEE Contr. Syst. Mag., vol. 11, no. 1, pp. 79-84, 1991.
[20] G. Stein, "Adaptive flight control-A pragmatic view," in Applicat. Adaptive Contr., K. S. Narendra and R. V. Monopoli, Eds. New York: Academic Press.

[21] C. Ling and T. F. Edgar, "A new fuzzy gain scheduling algorithm for process control," in Proc. Amer. Contr. Conf., Chicago, IL, 1992, vol. 4, pp. 2284-2290.

[22] B. C. Kuo and J. Tal, DC Motors and Control Systems. Champaign, IL: SRL Publishing, 1978.

[23] M. G. Say and E. O. Taylor, Direct Current Machines. New York: Wiley, 1980.

[24] B. Armstrong-Helouvry, "Stick slip and control in low-speed motion," IEEE Trans Automat. Contr., vol. 38, no. 10, pp. 1483-1496, 1993.

[25] P. E. DuPont, "Avoiding stick slip through PD control," IEEE Trans. Automat. Contr., vol. 39, no. 5, pp. 1094-1096, 1994

[26] R. C. Dorf, Modern Control Systems, Sixth Ed. Reading, MA: Addison-Wesley, 1992.

[27] K. J. Astrom and L. Rundqwist, "Integrator windup and how to avoid it," in Proc. Amer. Contr. Conf., vol. 2, 1989, pp. 1693-1698.

[28] B. Kosko, Neural Networks and Fuzzy Systems. Englewood Cliffs, NJ: Prentice-Hall, 1992.

[29] D. C. Montgomery, Design and Analysis of Experiments. New York: Wiley, 1991.

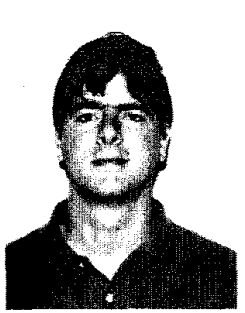

Jason T. Teeter received a BSEE degree from North Carolina State University in 1993 and is scheduled to receive a MSEE degreee from North Carolina State University in 1995.

$\mathrm{He}$ is currently a design engineer at GE Fanuc in Charlottesville, VA. His interests include artificial intelligence, embedded control system design and hardware design.

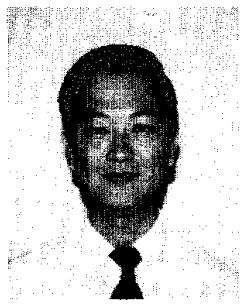

Mo-yuen Chow (S'81-M'82-M'83-S'83-M'87SM'93) earned the B.S. degree at the University of Wisconsin-Madison (1982); M.Eng. degree (1983), and Ph.D. degrees at Cornell University (1987), all in electrical engineering.

Upon completion of the Ph.D., he joined the faculty of North Carolina State University in Raleigh, NC, where he is presently an Associate Professor in the Department of Electrical and Computer Engineering. He has also worked as a consultant for Taiwan Power Company, J.W. Harley Company, and as a faculty intern at Duke Power Company. Since 1987, Dr. Chow has been working as a Principal Investigator in several projects in the areas of system monitoring, fault detection and control, applications of artificial neural networks and fuzzy logic. He has published over 40 technical papers related to his research work.

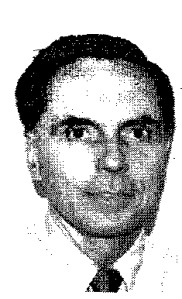

James J. Brickley Jr. (M'79) received the bachelors degree in mechanical engineering from the Stevens Institute of Technology in 1966, the M.S. degree in engineering mechanics from Virginia Polytechnic Institute and State University in 1972 and the Ph.D. degree in biomedical engineering from the University of Virginia in 1979.

$\mathrm{He}$ worked as a Research Engineer with the Naval Ship Research and Development Center in Washington, DC. He was Assistant Professor of Neurology and Neurosurgery and Research Associate Professor of Biomedical Engineering at the University of North Carolina at Chapel Hill and Administrative Director of the Clinical Neurophysiology Laboratory at North Carolina Memorial Hospital. He was Director of Engineering Research and Development at Isotechnologies, Inc. in Hillsborough, NC. He is currently Visiting Associate Professor and Associate Department Head in the Department of Electrical and Computer Engineering at North Carolina State University. 